\title{
Formation of $\omega$ Centauri from an Ancient Nucleated Dwarf Galaxy
}

\author{
Kenji Bekki \\ School of Physics, University of New South Wales, Sydney, NSW, 2052, \\ Australia \\ K. C. Freeman \\ Mount Stromlo and Siding Spring Observatories, The Australian \\ National University, Private Bag, P.O. Weston Creek, ACT 2611, \\ Australia
}

\begin{abstract}
We present a self-consistent dynamical model in which $\omega$ Cen is formed from an ancient nucleated dwarf galaxy merging with the first generation of the Galactic thin disc in a retrograde manner with respect to the Galactic rotation. Our numerical simulations demonstrate that during merging between the Galaxy and the $\omega$ Cen's host dwarf with $M_{\mathrm{B}} \simeq-14$ mag and its nucleus mass of $10^{7} M_{\odot}$, the outer stellar envelope of the dwarf is nearly completely stripped whereas the central nucleus can survive from the tidal stripping because of its compactness. The developed naked nucleus is orbiting the young Galactic disc in a retrograde manner with its apocenter and pericenter distances of $\sim 8 \mathrm{kpc}$ and $\sim 1 \mathrm{kpc}$, respectively, and thus have orbital properties similar to those of $\omega$ Cen. The Galactic tidal force can induce radial inflow of gas to the dwarf's center and consequently triggers moderately strong nuclear starbursts in a repetitive manner. This result implies that efficient nuclear chemical enrichment resulting from the later starbursts can be closely associated with the origin of the observed relatively young and metal-rich stars in $\omega$ Cen. Dynamical heating by the $\omega$ Cen's host can transform the young thin disc into the thick one during merging.
\end{abstract}

\section{Prediction of dynamical and chemical properties of the Galactic stellar halo formed from the $\omega$ Cen host.}

Since the details of the models and the results are given in Bekki \& Freeman (2003, MNRAS, 346, 11), only one important model prediction is described here. We predict that if $\omega$ Cen was previously the nucleus of a nucleated dwarf galaxy with orbital parameters given by Bekki \& Freeman (2003), the Galactic stellar halo stripped from the host shows crowding around $L_{\mathrm{z}} \sim-500$ and $L_{\mathrm{xy}} \sim 300$ $\mathrm{kpc} \mathrm{km} \mathrm{s}{ }^{-1}$, which reflects the orbital evolution of the dwarf. Here $L_{\mathrm{z}}$ represents angular momentum component in the $z$ direction and $L_{\mathrm{xy}}$ is $\left(L_{\mathrm{x}}^{2}+L_{\mathrm{y}}^{2}\right)^{1 / 2}$. 